\title{
Antabuse for Lyme Disease: The Way Forward
}

\author{
David S. Younger, Beverley F. Murphy \\ Department of Clinical Medicine, CUNY School of Medicine, City College, New York, USA \\ Email: youngd01@nyu.edu
}

How to cite this paper: Younger, D.S. and Murphy, B.F. (2020) Antabuse for Lyme Disease: The Way Forward. World Journal of Neuroscience, 10, 1-7.

https://doi.org/10.4236/wjns.2020.101001

Received: November 18, 2019

Accepted: November 26, 2019

Published: November 29, 2019

Copyright $\odot 2020$ by author(s) and Scientific Research Publishing Inc. This work is licensed under the Creative Commons Attribution International License (CC BY 4.0).

http://creativecommons.org/licenses/by/4.0/

\begin{abstract}
Despite leadership by dedicated lay organization and patient-based advocacy groups, the way forward for prospective candidates for disulfiram treatment has been difficult. This article provides the background, overview of disulfiram, in particular its intended use in post-treatment Lyme disease syndrome after a standard of care course of antibiotics fails to alleviate symptoms of tick borne disease. A series of recommendations are offered to guide patients and clinicians.
\end{abstract}

\section{Keywords}

Disulfiram, Antabuse, Lyme Disease, Immune Globulin Therapy

\section{Background and Overview}

Borrelia (B.) burgdorferi sensu lato is the causative agent of Lyme disease worldwide, while the genus, $B$. burgdorferi sensu stricto is the causal infectious agent of the North American disease transmitted by the bite of hard-shelled Ixodes scapularis ticks. Understanding the biology of $B$. burgdorferi notwithstanding other vectors including B. duttonii, B. garinii, B. afzelii, and B. miyamotoi [1] has been important in applied clinical research in devising prevention and treatment strategies.

With or without known tick attachment, the common presenting clinical findings of Lyme disease include erythema migrans (EM), fatigue, fever, chills, muscle, and joint pain [2]. However they too may be absent or overlooked leading to a delay in serological diagnosis and effective treatment. In the absence of effective antibiotic therapy, B. burgdorferi affects immunocompetent hosts, heightening the immune response [3] with a propensity to establish long-lasting infection in its natural (white footed mice) [4] [5] and incidental (humans) hosts [6] [7]. In the nervous system experimental B. burgdorferi inoculation in rhesus macaque primates induces inflammatory mediators in glial and neuronal cells 
that precipitate glial and neuronal apoptosis and an array of neurologic changes recreating the clinical, serologic, and neuropathologic picture of human Lyme disease [3].

The recurrence or emergence of systemic and nervous system symptoms after a standard of care course of therapy may occur with an enduring positive or indeterminate two-tier Lyme serology or other biomarkers in the cerebrospinal fluid (CSF), and abnormal findings in neuroimaging, electrodiagnostic and autonomic studies correlative with central, peripheral and autonomic nervous system (CNS, PNS and ANS) symptoms and signs [8] [9] have led to reconsideration of post-treatment Lyme disease syndromes (PTLDS).

It is not well understood why some patients experience PTLDS, however the varied presentation no doubt incorporates at least four etiopathogenic mechanisms, singly or in combination in affected cases including persistent but difficult to detect infection [10], host-induced immunity targeting non-viable spirochetes or their remnants [11], a triggered immune response that lasts well after the infection similar to Group A beta hemolytic streptococcus (GABHS) infection in rheumatic heart disease and pediatric autoimmune neuropsychiatric disorder (PANDAS) [12] [13]; and as some might argue, causes unrelated to infection or immunity [14].

The antibiotic treatment of patients with PTLDS has been stymied by negative randomized placebo-controlled trials that fail to show significant, sustained benefit of antibiotic retreatment [15] [16], yet this may be related in part to the antibiotic regimens used, the limitations of current antibody-based assays, polymerase chain reaction (PCR), and culture techniques to determine successful eradication of $B$. burgdorferi.

\section{Disulfiram for PTLDS}

Among highly active compounds investigated in vitro with high-throughput screening of four compound libraries (Library of Pharmacologically Active Compounds, the National Institutes of Health Clinical Collection, the Microsource Spectrum, and the Biomol Food and Drug Administration [FDA]) to inhibit $>90 \%$ of B. burgdorferi growth at a concentration of $<25 \mu \mathrm{M}$ [17], disulfiram (Antabuse ${ }^{\oplus}$ ) emerged in 2016 as a lead candidate based upon a favorable minimum inhibitory concentration and minimum bactericidal concentration against $B$. burgdorferi in vitro. The narrow antibacterial spectrum of disulfiram-based disulfides derived from disulfiram was later appreciated [18].

Disulfiram is an oral prescription drug for the treatment of alcohol abuse disorders [19]. Upon absorption, disulfiram and its metabolites inhibit aldehyde dehydrogenase enzymes that oxidize acetaldehyde from ethanol metabolism into acetic acid. The inactivation of hepatic ALDH leads to buildup of toxic acetaldehyde in the body, which manifests "hangover" symptoms of headache and nausea that deters alcohol consumption [20]. Disulfiram is readily cleaved by thiol-bearing substances such as cysteine enzymes which result in the simulta- 
neous addition and release of diethyldithiocarbamate (DDC) which potentiates the antibacterial activity of disulfiram as both a standalone and combination bactericidal agent [21]. Diethyldithiocarbamate is a very strong chelator of transition divalent metal ions, mainly copper (II) $(\mathrm{Cu})$, and the in vitro cytotoxicity of disulfiram is $\mathrm{Cu}$-dependent. When DDC contacts $\mathrm{Cu}$, the chelating reaction between them triggers the generation of reactive oxygen species (ROS) [22], which damage DNA, protein and lipids leading to cell death. ROS are extremely transient species with very short lifetime due to their high chemical reactivity and can only penetrate very short distance in tissues [23]. Moreover, the reaction of disulfiram and $\mathrm{Cu}$ must take place inside or adjacent to the target cells. In addition to ROS generated from $\mathrm{DDC}$ and $\mathrm{Cu}$ reaction, bio ( $\mathrm{N}, \mathrm{N}$-diethyldithiocarbamato) copper (II) (Cu-DDC), the end product derived from DDC and $\mathrm{Cu}$ reaction, is also cytotoxic [24] [25].

This year, 3 patients who participated in a uncontrolled open-label trial of 500 mg of disulfiram daily for symptoms of chronic relapsing neurological Lyme disease and relapsing babesiosis were able to discontinue treatment and remain clinically well for 6 to 23 months [26]. An initial study (ClinicalTrials.gov Identifier: NCT03891667) of the side effects, tolerability and initial signs of the effectiveness of disulfiram in reducing symptoms among the 24 patients with PTLDS is actively recruiting subjects. Two proposed parallel treatment schedules will be compared. In the experimental arm of the study, one-half of subjects will get 8 weeks of two $250 \mathrm{mg}$ of daily disulfiram with active comparison to another study arm of a shorter duration of disulfiram for 4 weeks followed by 4 weeks of matching placebo capsules. Primary and secondary outcome measures assessed over a 10 week period are carried out with psychometrically validated forms, respectively employing Fatigue Severity Scale (FSS) and Quality of Life Enjoyment and Satisfaction Questionnaire-Short Form (Q-LES-Q-SF); and Short Form Health Survey (SF-36), General Symptom Questionnaire (GSQ-30); and PROMIS-29 surveys. Commensurate with its possible drug toxicity, subjects with existing seizure disorder, renal and liver disease, and peripheral neuropathy are excluded from study participation. The starting dose of $500 \mathrm{mg}$ daily is higher than the recommended stating daily dose of $250 \mathrm{mg}$ used by addiction psychiatrists to treat alcohol addiction, and other physicians treating Lyme disease who administer a starting dose of $62.5 \mathrm{mg}$ and slowly increase to the target dose of $250 \mathrm{mg}$ daily.

\section{Side Effects and Contraindications}

Concern for the development of peripheral neuropathy so noted anecdotally in up to a third of patients with PTLDS treated with disulfiram had led to concern regarding the initiation of treatment in those with pre-existing neuropathy and the most appropriate manner of screening patients who may be prone to, or have existing neuropathy. Caution dictates that prior to start of disulfiram therapy, candidates with PTLDS be screened with a careful history, clinical neurological examination and three limb electrodiagnostic studies to delineated a large 
fiber polyneuropathy; and intraepidermal nerve fiber (IENF) analysis of a calf and thigh via $3 \mathrm{~mm}$ punch skin biopsy site for density measures to disclose small fiber sensory polyneuropathy. The latter examination correlates well with painful peripheral neuropathy [27]. Peripheral neuropathy may be due in part to the DDC Cu complex leading some to consider monitoring of copper levels and using chelation. However, more likely it results from a combination of factors that impact the dorsal root ganglion neuron and its peripheral processes that include direct neurotoxicity of disulfiram or its metabolites, concomitant medications in a given patient, and the underlying predisposition for neuropathy based upon a post-infectious immune response associated with PTLDS and tick borne disease coinfections. The existence of peripheral neuropathy is not an absolute exclusion for disulfiram therapy if there is ongoing active treatment thereof such as intravenous immune globulin (IVIg) therapy which may have both prophylactic and efficacy in emergent cases.

Neuropyshciatric disturbances notably depression and psychosis [27] may potentially occur. Serum and CSF measures of DBH have reported usefulness in predicting the increased risk of such side effects [28]. Patients with new onset or exacerbation of neuropsychiatric disturbances should not be continued on disulfiram.

\section{Impact of Social Media}

Over 9000 individuals have joined one of several Facebook groups (Disulfiram for Lyme Support Group (Antabuse); Disulfiram/Antabuse for Lyme; Disulfiram/Antabuse Lyme Success Stories; Disulfiram for Lyme Group; Our Journeys: Disulfiram and Tick Borne Disease; Youth Disulfiram Lyme Support (Antabuse)) to exchange anecdotal information of their experience and questions regarding disulfiram in the treatment of Lyme disease. Forum members report an excess of fatigue, body pain, nausea, headaches, painful peripheral neuropathy and neuropsychiatric disturbances. However, these are uncontrolled observations and they may reflect a combination of dose-related and idiosyncratic drug side effects, as well as exacerbation of PTLDS complaints and unrelated pre-existing disturbances. Notwithstanding, the emergence of side effects has escalated caution as to the correct approach to the start, continuation and discontinuation of therapy, and the necessary duration of treatment in appropriate patients.

Despite leadership by dedicated lay organization and patient-based advocacy groups, the way forward for prospective candidates for disulfiram treatment has been difficult in the absence of leadership by accredited physician and university program leadership.

\section{Recommendations}

It is possible to forward several recommendations based upon the current knowledge base. 
1) Awaiting the results of clinical trials, individuals with PTLDS should consider treatment with disulfiram as an adjunct to conventional antibiotics but not a replacement.

2) Physician prescribed treatment with disulfiram should be carried out employing standard doses of $250 \mathrm{mg}$ (or fractions thereof).

3) Safety monitoring should include blood studies for systemic complications, and avoidance of contact with alcohol in toiletries, foods, beverages, medications, and other sources of inadvertent alcohol exposure.

4) A starting dose as low as $62.5 \mathrm{mg}$ can be slowly increased to the target dose depending upon tolerability and side effects.

5) Common side effects that may limit achievement of full doses include fatigue, body pain, nausea, headaches, peripheral neuropathy and neuropsychiatric disturbances.

6) The duration of treatment should be carefully determined among all individuals in keeping with empiric therapy to avoid inadvertent toxicity or prolonged use of disulfiram.

7) Peripheral neuropathy is not an absolute exclusion for consideration of disulfiram. Such cases should be carefully examined at baseline and monitored in the course of therapy.

8) Cases of painful small fiber sensory polyneuropathy may be monitored with IENF analysis in a punch skin biopsy of the calf and thigh.

9) IVIg may be initiated before disulfiram in existing cases of peripheral neuropathy as an effective treatment, or in the course of therapy for those with emergent neuropathy of either large or small fiber caliber, or exacerbation of either pre-existing disease.

10) Emergency or exacerbation of neuropsychiatric disturbances should lead to discontinuation of medication.

11) One or more registries should be created on social media with anonymous participation to gather information on the use of disulfiram in PTLDS.

12) Physicians should also consider establishing a registry with anonymous participation to follow emerging trends in the epidemiology of disulfiram in the PTLDS population.

\section{Conflicts of Interest}

The authors declare no conflicts of interest regarding the publication of this paper.

\section{References}

[1] Brownstein, J.S., Holford, T.R. and Fish, D. (2005) Effect of Climate Change on Lyme Disease Risk in North America. Ecohealth, 2, 38-46.

https://doi.org/10.1007/s10393-004-0139-x

[2] Borchers, A.T., Keen, C.L., Huntley, A.C., et al. (2015) Lyme Disease: A Rigorous Review of Diagnostic Criteria and Treatment. Journal of Autoimmunity, 57, 82-115. https://doi.org/10.1016/j.jaut.2014.09.004 
[3] Ramesh, G., Didier, P.J., England, J.D., et al. (2015) Inflammation in the Pathogenesis of Lyme Neuroborreliosis. The American Journal of Pathology, 185, 1344-1360. https://doi.org/10.1016/j.ajpath.2015.01.024

[4] Barthold, S.W., de Souza, M.S., Janotka, J.L., et al. (1993) Chronic Lyme Borreliosis in the Laboratory Mouse. The American Journal of Pathology, 143, 959-971.

[5] Hodzic, E., Feng, S., Holden, K., et al. (2008) Persistence of Borrelia burgdorferi Following Antibiotic Treatment in Mice. Antimicrobial Agents and Chemotherapy, 52, 1728-1736. https://doi.org/10.1128/AAC.01050-07

[6] Steere, A.C., Schoen, R.T. and Taylor, E. (1987) The Clinical Evolution of Lyme Arthritis. Annals of Internal Medicine, 107, 725-731. https://doi.org/10.7326/0003-4819-107-5-725

[7] Diterich, I., Rauter, C., Kirschning, C.J., et al. (2003) Borrelia burgdorferi-Induced Tolerance as a Model of Persistence via Immunosuppression. Infection and Immunity, 71, 3979-3987. https://doi.org/10.1128/IAI.71.7.3979-3987.2003

[8] Younger, D. (2019) Serial Brain Positron Emission Tomography Fused to Magnetic Resonance Imaging in Post-Infectious and Autoantibody-Associated Autoimmune Encephalitis. World Journal of Neuroscience, 9, 153-156.

https://doi.org/10.4236/wjns.2019.93010

[9] Younger, D.S., Wu, W.E., Hardy, C., et al. (2012) Lyme Neuroborreliosis and Proton MR Spectroscopy: Preliminary Results from an Urban Referral Center Employing Strict CDC Criteria for Case Selection. Neurology, 78, PO3.246.

https://doi.org/10.1212/WNL.78.1_MeetingAbstracts.P03.246

[10] Marques, A., Telford, S.R., Turk, S.P., et al. (2014) Xenodiagnosis to Detect Borrelia burgdorferi Infection: A First-in-Human Study. Clinical Infectious Diseases, 58, 937-945. https://doi.org/10.1093/cid/cit939

[11] Coughlin, J.M., Yang, T., Rebman, A.W., et al. (2018) Imaging Glial Activation in Patients with Post-Treatment Lyme Disease Symptoms: A Pilot Study Using [11C]DPA-713 PET. Journal of Neuroinflammation, 15, 346. https://doi.org/10.1186/s12974-018-1381-4

[12] Younger, D.S. (2019) The Autoimmune Brain. Rowman and Littlefied, Lanham.

[13] Younger, D.S. and Bouboulis, D.A. (2015) Immune Pathogenesis of Pediatric Autoimmune Neuropsychiatric Disorders Associated with Group A $\beta$-Hemolytic Streptococcal Infections (PANDAS). International Journal of Neurology Research, $1,5-7$.

[14] Feder, H.M., Johnson, B.J.B., O’Connell, S., et al. (2007) A Critical Appraisal of Chronic Lyme Disease. The New England Journal of Medicine, 357, 1422-1430. https://doi.org/10.1056/NEJMra072023

[15] Klempner, M.S., Hu, L.T., Evans, J., et al. (2001) Two Controlled Trials of Antibiotic Treatment in Patients with Persistent Symptoms and a History of Lyme Disease. The New England Journal of Medicine, 345, 85-92. https://doi.org/10.1056/NEJM200107123450202

[16] Fallon, B.A., Keilp, J.G., Corbera, K.M., et al. (2008) A Randomized, Placebo-Controlled Trial of Repeated IV Antibiotic Therapy for Lyme Encephalopathy. Neurology, 70, 992-1003. https://doi.org/10.1212/01.WNL.0000284604.61160.2d

[17] Pothineni, V.R., Wagh, D., Babar, M.M., et al. (2016) Identification of New Drug Candidates against Borrelia burgdorferi Using High-Throughput Screening. Drug Design, Development and Therapy, 10, 1307-1322.

https://doi.org/10.2147/DDDT.S101486 
[18] Sheppard, J.G., Frazier, K.R., Saralkar, P., et al. (2018) Disulfiram-Based Disulfides as Narrow-Spectrum Antibacterial Agents. Bioorganic \& Medicinal Chemistry Letters, 28, 1298-1302. https://doi.org/10.1016/j.bmcl.2018.03.023

[19] Ellis, P.M. and Dronsfield, A.T. (2013) Antabuse's Diamond Anniversary: Still Sparkling on? Drug and Alcohol Review, 32, 342-344. https://doi.org/10.1111/dar.12018

[20] Wright, C. and Moore, R.D. (1990) Disulfiram Treatment of Alcoholism. The American Journal of Medicine, 88, 647-655. https://doi.org/10.1016/0002-9343(90)90534-K

[21] Frazier, K.R., Moore, J.A. and Long, T.E. (2019) Antibacterial Activity of Disulfiram and Its Metabolites. Journal of Applied Microbiology, 126, 79-86. https://doi.org/10.1111/jam.14094

[22] Lewis, D.J., Deshmukh, P., Tedstone, A.A., et al. (2014) On the Interaction of Copper (II) with Disulfiram. Chemical Communications, 50, 13334-13337. https://doi.org/10.1039/C4CC04767B

[23] D’Autreaux, B. and Toledano, M.B. (2007) ROS as Signaling Molecules: Mechanisms That Generate Specificity in ROS Homeostasis. Nature Reviews Molecular Cell Biology, 8, 813-824. https://doi.org/10.1038/nrm2256

[24] Tawari, P.E., Wang, Z., Najlah, M., et al. (2015) The Cytotoxic Mechanisms of Disulfiram and Copper (II) in Cancer Cells. Toxicological Research, 4, 1439-1442. https://doi.org/10.1039/C5TX00210A

[25] Valentine, W.M. (2019) Toxic Peripheral Neuropathies: Agents and Mechanisms. Toxicologic Pathology. https://doi.org/10.1177/0192623319854326

[26] Liegner, K.B. (2019) Disulfiram (Tetraethylthiuram Disulfide) in the Treatment of Lyme Disease and Babesiosis: Report of Experience in Three Cases. Antibiotics (Basel), 8, 72. https://doi.org/10.3390/antibiotics8020072

[27] Oaklander, A.L. (2015) Immunotherapy Prospects for Painful Small-Fiber Sensory Neuropathies and Ganglionopathies. Neurotherapeutics, 13, 108-117. https://doi.org/10.1007/s13311-015-0395-1

[28] Major, L.F., Lerner, P., Ballenger, J.C., et al. (1979) Dopamine-Beta-Hydroxylase in the Cerebrospinal Fluid: Relationship to Disulfiram-Induced Psychosis. Biological Psychiatry, 14, 337-344. 